\title{
Three-Particle Recombination at Low Temperature: a QED Approach
}

\author{
S. BhatTACHARYYA* AND A. Roy \\ Department of Physics, Gokhale Memorial Girls' College \\ Calcutta-700020, India
}

(Received February 26, 2001; revised version May 23, 2001)

\begin{abstract}
A theoretical study of the three-body recombination of proton and electron in presence of a spectator electron with electronic beam at near-zero temperature is presented using field theory and invariant Lorentz gauge. Contributions from the Feynman diagrams of different orders give an insight into the physics of the phenomena. Recombination rate coefficient is obtained for low lying principal quantum number $n=1$ to 10 . At a fixed ion beam temperature $(300 \mathrm{~K})$ recombination rate coefficient is found to increase in general with $n$, having a flat and a sharp peak at quantum states 3 and 5, respectively. In absence of any other theoretical and experimental results for low temperature formation of $\mathrm{H}$-atom by three-body recombination at low lying quantum states, we have presented the theoretical results of Stevefelt and group for three-body recombination of deuteron with electron along with the present results. Three-body recombination of antihydrogen in antiproton-positron plasma is expected to yield similar result as that for three-body recombination of hydrogen formation in proton-electron plasma. The necessity for experimental investigation of low temperature three-body recombination at low quantum states is stressed.
\end{abstract}

PACS numbers: 32.80.Fb, 33.55.Ad

\section{Introduction}

When electron combines with atomic ion in absence of any third body, the energy which is liberated is carried off as radiation. This type of radiative recom-

*corresponding author; e-mail: sbgcmr@cal2.vsnl.net.in 
bination (RR) occurs in thin plasma. In the case of dense plasma, part of the energy liberated may be given to a neighbouring electron. This is collisional recombination or three-body recombination (TBR). In the dense plasma TBR is one of the major causes of loss of free electrons. If the temperature of the ions is such that the mean thermal energy is much smaller than the first excitation energy of the ion-electron bound state, radiative recombination becomes slow but excited hydrogenic ions are rapidly formed by TBR.

The radiative transition rates for hydrogen atom have been extensively calculated on the basis of quantum mechanics [1]. In very tenuous plasma, recombination will proceed by radiative electron capture, preferentially into lower quantum levels. This radiative recombination has been theoretically studied by many authors [2-6]. In laboratory plasmas, however, energy exchanges through electron collisions are expected to play an important role as well. In heavy ion cooler rings the phase space cooling of ion beams have been realized by merging the heavy-ion beams with dense and very cold electron beams at the cooler. When cooling bare ions with electrons, recombination processes between them provide the main decay channel in a ring. Theoretical models, describing recombination of bare ions with electron, combine two basic processes, namely RR and TBR. The TBR has recently gained increased interest as a promising mechanism for antihydrogen production in ion traps [7, 8]. Beyer et al. [9] have suggested that TBR may play a role in electron cooler rings. Recently, electron cooler beams of very low temperatures have been produced through an adiabatic beam expansion technique. Theoretical models for TBR [10,11] predict that mainly high lying Rydberg states are populated and that its total rate depends strongly on plasma temperature and Saha equilibrium electron density. At low equivalent plasma temperatures available at electron cooler, TBR becomes comparable to $\mathrm{RR}$ in populating $n>15$ Rydberg states. Several experiments have addressed these issues by measuring rate coefficients through the detection of recombined particles [12-17]. These measurements are sensitive mostly to low $n$ states of ions and many of them report higher recombination rates than could be accounted for by radiative recombination theory. To explain the discrepancy between theory and experiment in the RR process one of the authors (S.B.) have conjectured a second channel [5a], namely, two-step radiative recombination (TSRR) channel over and above the spontaneous RR channel [6]. However, the observed enhancement of ion-electron recombination rate for bare ions is yet poorly understood. Several conditions, ranging from TBR [13] to the effect of interaction field in the interaction region [18], have been proposed as a possible case of the observed enhanced rates. Since TBR is thought to lead mainly to the population of high $n$ Rydberg states, attempts were made at studying the population of such states via recombination [19]. Quinteros et al. [17] investigated spontaneous electron-ion recombination mechanism at near-zero relative velocities. In early 1960s Bates et al. suggested that it is appropriate to consider electron-ion recombination processes as components of a collision-radiative 
complex model $[2,10]$. This model predicted that the TBR process may play an important role in low temperature dense plasma. Stevefelt et al. employed classical trajectory Monte Carlo calculation to derive simple analytical formula for TBR. TBR rates of $\mathrm{D}^{+}$with electron are calculated by Stevefelt et al. [10,11]. In Bates et al. approach [10] TBR diverges for high $n$ as $n^{4}$. Bates et al. model differs from that of Stevefelt et al. by predicting a temperature dependence $\propto T^{-2}$. Thus we find that low temperature TBR phenomena are not yet clearly understood both at low and high $n$ states. Since there is still scope for theorisation, we use quantum electrodynamics (QED) to compute TBR. Feynman diagrams, covariant Lorentz gauge, and QED have previously successfully explained various bound state collision phenomena [20-22]. As such, we are tempted to apply QED to study TBR.

The four lowest order Feynman diagrams contributing to TBR are shown in Figs. 1a-d. Using the nomenclature from previous works [20, 21] we can identify two mechanisms in TBR, such as shake-in (SI) mechanisms and two-step (TS) mechanisms. The two second-order Feynman diagrams (Figs. 1a,b) are due to SI processes being named as SI-1 and SI-2, respectively. In SI-1, one of the electrons after being scattered by the spectator electron is attracted towards the bare ion and forms bound state with the ion. In SI-2 process it is the bare ion which scatters the spectator electron. Due to change in the Coulomb field of the ion the second electron is attracted towards the ion to form bound state. The two fourth-order Feynman diagrams (Figs. 1c,d) represent TS mechanisms, being named respectively as TS-1a and TS-1b. In TS-1a process (Fig. 1c) the spectator electron is at first scattered due to exchange of virtual photon with the second electron which in turn emits another photon to be absorbed by the bare ion and ion-electron recombination takes place at various quantum states. In TS-1b process (Fig. 1d), at first virtual photon exchange occurs between the ion and the spectator electron which is scattered. The ion subsequently emits a second photon, which the neighbouring electron absorbs and gets bound to the ion.
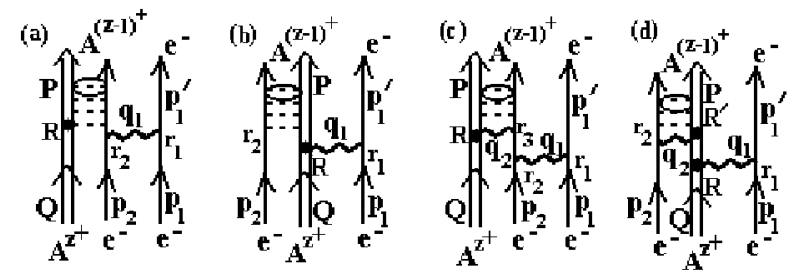

Fig. 1. Feynman diagram for three-body recombination of proton with electron in presence of a spectator electron. Double lines represent bare ions and single lines represent electrons. The wavy lines represent transverse photon exchange. The dashed lines represent Coulomb photon exchange in the bound state. Shake-in mechanism: (a) SI-1, (b) SI-2. Two-step-one mechanism: (c) TS-1a, (d) TS-1b. 


\section{Theoretical formalism}

In QED, a composite system of bound particles are represented by a string of field operators operating on particle vacuum and multiplied by unperturbed solution of Schrödinger equation [23]. The creation and annihilation operators of the particles bound to the Coulomb field of the nucleus obey free field equal-time commutation relations. There will be no loss of generality to represent the bound particles by the Feynman directed lines. An odd half spin bare ion can also be represented by the Feynman directed line [24]. The Feynman diagrams of appropriate order are computed to study TBR reaction

$$
\mathrm{A}^{Z^{+}}+\mathrm{e}^{-}+\mathrm{e}^{-} \rightarrow\left(\mathrm{A}^{(Z-1)^{+}}\right)^{*}+\mathrm{e}^{-}
$$

where $\mathrm{A}^{Z^{+}}$is an odd half spin bare ion of atomic number $Z$.

In the second-order SI processes recombination occurs due to change in the Coulomb field of the ion. In the fourth-order TS processes one of the electrons gets scattered either due to electron-electron interaction (Fig. 1c) or due to electron-ion interaction (Fig. 1d), while the capture of the second electron occurs due to exchange of a transverse photon between the ion and the second electron. We find, in the case of TS-1a and TS-1b, the particle propagators are, respectively, electron propagator and ion propagator. Although we have restricted ourselves to low-order perturbation theory with regard to radiation field (orders $e^{2}$ and $e^{4}, S$-matrices), we can obtain results accurate to all orders in the Coulomb coupling by suitable choice of the wave functions. The Coulomb distortion factor is included in the wave function to estimate this effect of the Coulomb coupling over the $S$-matrix particle-radiation field coupling. To write $S$-matrices for reaction (1) from Feynman diagrams (Figs. 1a-d) we define the following terms.

\subsection{Definition}

4-momenta of the initial ion and two electrons are, respectively, $Q\left(Q_{0}, \boldsymbol{Q}\right)$, $p_{1}\left(p_{10}, \boldsymbol{p}_{1}\right)$, and $p_{2}\left(p_{20}, \boldsymbol{p}_{2}\right)$ and after recombination 4 -momenta of the ion, scattered electron and bound electron are, respectively, $P\left(P_{0}, \boldsymbol{P}\right), p_{1}^{\prime}\left(p_{10}^{\prime}, \boldsymbol{p}_{1}^{\prime}\right)$, and $b\left(b_{0}, b\right)$.

$r_{1}\left(r_{10}, r_{1}\right), r_{2}\left(r_{20}, r_{2}\right)$, and $R\left(R_{0}, \boldsymbol{R}\right)$ are the 4 -coordinates of the two electrons and ion, respectively, and $R^{\prime}\left(R_{0}^{\prime}, \boldsymbol{R}^{\prime}\right)$ is the 4 -coordinate of the final ion in Fig. 1d. The wave function of the electron is given by [25]:

$$
\left[\psi_{\mathrm{e}}\left(p_{i}\right)\right]_{r_{i}}=F_{\mathrm{C}}\left(r_{i}, p_{i}\right) \sqrt{\frac{1}{V(2 \pi)^{3}}} \sqrt{\frac{m}{E_{p_{i}}}} u\left(p_{i}\right) \mathrm{e}^{\mathrm{i} p_{i} r_{i}}, \quad i=1,2,
$$

where the Coulomb distortion factor

$$
F_{\mathrm{C}}\left(r_{i}, p_{i}\right)=\mathrm{e}^{-\mathrm{i} \eta_{i} / 2} \Gamma\left(1+\mathrm{i} \eta_{i}\right)_{1} F_{1}\left[-\mathrm{i} \eta_{i}, 1, \mathrm{i}\left(\left|p_{i}\right|\left|r_{i}\right|-p_{i} \cdot r_{i}\right)\right]
$$




$$
\eta_{i}=\frac{[m M /(m+M)] e^{2} Z}{\hbar^{2}\left|p_{i}\right|},
$$

$m$ and $M$ are the masses of electron and bare ion, respectively; $V$ is the volume, $u\left(p_{i}\right)$ is electron spinor.

The relativistic energy of incident and scattered electrons are, respectively

$$
\begin{aligned}
& E_{p_{i}}=p_{i 0}=m c^{2}+E_{i}, \\
& E_{p_{1}^{\prime}}=p_{10}^{\prime}=m c^{2}+E_{1}^{\prime},
\end{aligned}
$$

where $E_{i}$ are the kinetic energies of the two incident electrons and $E_{1}^{\prime}$ is the kinetic energy of the scattered electron. The wave function for the bound electron in $(n, l)$ state is given by [23]:

$$
\left[\psi_{e}(b)\right]_{r_{i}}=\sqrt{\frac{1}{V(2 \pi)^{3}}} \sqrt{\frac{m}{E_{b}}} V\left(b, r_{i}\right) \phi_{n l}(x) .
$$

$V\left(b, r_{i}\right)$ is spinor of a bound electron given by

$$
V\left(b, r_{i}\right)=\left(1-\frac{1}{2} \alpha Z \gamma_{4} \gamma \cdot n\right)(2 \pi)^{-3 / 2} u(b) \mathrm{e}^{\mathrm{i} b r_{i}}
$$

where $n=\frac{\boldsymbol{r}_{i}}{\left|\boldsymbol{r}_{i}\right|}, \alpha=\frac{1}{137}, \gamma\left(\gamma_{1}, \gamma_{2}, \gamma_{3}\right)$ is the Dirac $\gamma$-matrix.

In our computation we shall consider only the first term of the expansion ( 7 ) since the second term is small of the order $\alpha . E_{\mathrm{b}}$ is the relativistic energy of bound electron,

$$
E_{\mathrm{b}}=b_{0}=m c^{2}-\varepsilon,
$$

where $\varepsilon$ is the binding energy of the electron, $\phi_{n l}(x)$ is the Schrödinger solution for one-electron ion,

$$
x=r_{2}-\boldsymbol{R} .
$$

The wave function for the incident ion is

$$
\psi_{\mathrm{A}^{z}+}(Q)=\sqrt{\frac{1}{V(2 \pi)^{3}}} \sqrt{\frac{M}{E_{Q}}} U(Q) \mathrm{e}^{\mathrm{i} Q R} .
$$

The relativistic energy of incident ion is given by

$$
E_{Q}=Q_{0}=M c^{2}+E,
$$

where $E$ is the kinetic energy of the incident ion, $M$ is the mass of the ion, $U(Q)$ is the spinor of the incident ion. The wave function of the ion after recombination is given by

$$
\psi_{\mathrm{A}^{Z}}(P)=\sqrt{\frac{1}{V(2 \pi)^{3}}} \sqrt{\frac{M}{E_{P}}} U(P) \mathrm{e}^{\mathrm{i} P R} .
$$

The relativistic energy of the ion after recombination is $E_{P}=P_{0}=M c^{2}+E^{\prime}$, where $E^{\prime}$ is the kinetic energy of the ion after recombination, $U(P)$ is the spinor 
of the ion after recombination. The photon propagator between $r_{1}$ and $r_{2}$ is given by

$$
D\left(r_{2}-r_{1}\right)=\frac{1}{(2 \pi)^{4}} \int \frac{\mathrm{e}^{\mathrm{i} q_{1}\left(r_{2}-r_{1}\right)}}{q_{1}^{2}+\mathrm{i} \epsilon} \mathrm{d}^{4} q_{1}
$$

The electron propagator between $r_{3}$ and $r_{2}$ is defined as

$$
S\left(r_{3}-r_{2}\right)=\frac{1}{(2 \pi)^{4}} \int \frac{\mathrm{e}^{\mathrm{i} s\left(r_{3}-r_{2}\right)}}{\hat{s}-m} \mathrm{~d}^{4} s=\frac{1}{(2 \pi)^{4}} \int \frac{\mathrm{e}^{\mathrm{i} s\left(r_{3}-r_{2}\right)}(\hat{s}+m)}{s^{2}-m^{2}} \mathrm{~d}^{4} s,
$$

where

$$
\widehat{s}=s \gamma
$$

The ion propagator between $R^{\prime}$ and $R$ is given by

$$
S_{i}\left(R^{\prime}-R\right)=\frac{1}{(2 \pi)^{4}} \int \frac{\mathrm{e}^{\mathrm{i} l\left(R^{\prime}-R\right)}}{\widehat{l}-m} \mathrm{~d}^{4} l=\frac{1}{(2 \pi)^{4}} \int \frac{\mathrm{e}^{\mathrm{i} l\left(R^{\prime}-R\right)}(\widehat{l}+m)}{l^{2}-M^{2}} \mathrm{~d}^{4} l,
$$

where

$$
\widehat{l}=l \gamma
$$

The electron current is given by

$$
J_{\mathrm{e}}^{\mu}\left(p_{i}^{\prime}, p_{i}, r_{i}\right)=\frac{-\mathrm{i} e}{\sqrt{\hbar c}}\left[\bar{\psi}_{\mathrm{e}}\left(p_{i}^{\prime}\right) \gamma_{\mu} \psi_{\mathrm{e}}\left(p_{i}\right)\right]_{r_{i}} .
$$

The ion current is

$$
J_{\mathrm{A}^{Z+}}^{\mu}(P, Q, R)=\frac{\mathrm{i} e Z}{\sqrt{\hbar c}}\left[\bar{\psi}_{\mathrm{A}^{z}+}(P) \gamma_{\mu} \psi_{\mathrm{A}^{z}+}(Q)\right]_{R} .
$$

Let the $S$-matrices corresponding to Figs. 1a-d be respectively $S_{1}, S_{2}, S_{3}$, and $S_{4}$ and are given below

$$
\begin{aligned}
S_{1}= & J_{\mathrm{e}}^{\mu}\left(p_{1}^{\prime}, p_{1}, r_{1}\right) D\left(r_{2}-r_{1}\right) J_{\mathrm{e}}^{\nu}\left(b, p_{2}, r_{2}\right)\left[\psi_{\mathrm{A}^{z}}(P) \psi_{\mathrm{A}^{z}}(Q)\right]_{R}, \\
S_{2}= & J_{\mathrm{e}}^{\mu}\left(p_{1}^{\prime}, p_{1}, r_{1}\right) D\left(R-r_{1}\right) J_{\mathrm{A}^{z+}}^{\nu}(P, Q, R)\left[\bar{\psi}_{\mathrm{e}}(b) \psi_{\mathrm{e}}\left(p_{2}\right)\right]_{r_{2}}, \\
S_{3}= & J_{\mathrm{e}}^{\mu}\left(p_{1}^{\prime}, p_{1}, r_{1}\right) D\left(r_{2}-r_{1}\right)\left(\frac{-\mathrm{i} e}{\sqrt{\hbar c}}\right)^{2} \\
& \times\left[\bar{\psi}_{\mathrm{e}}\left(b, r_{3}\right) \gamma_{\nu} S\left(r_{3}-r_{2}\right) \gamma_{\delta} \psi_{\mathrm{e}}\left(p_{2}, r_{2}\right)\right] D\left(R-r_{3}\right) J_{\mathrm{A}^{z+}}^{\rho}(P, Q, R), \\
S_{4}= & J_{\mathrm{e}}^{\mu}\left(p_{1}^{\prime}, p_{1}, r_{1}\right) D\left(R-r_{1}\right)\left(\frac{\mathrm{i} Z e}{\sqrt{h c}}\right)^{2} \\
& \times\left[\bar{\psi}_{\mathrm{A} z}+(P) \gamma_{\nu} S_{i}\left(R^{\prime}-R\right) \gamma_{\delta} \psi_{\mathrm{A} z}(Q)\right]_{R, R^{\prime}} D\left(r_{2}-R^{\prime}\right) J_{\mathrm{e}}^{\rho}\left(b, p_{2}, r_{2}\right) .
\end{aligned}
$$




\subsection{Cross-section}

The matrix element for the process (1) is the sum of the contributions from all the diagrams as given below

$$
\left|M_{f i}\right|=S_{1}+S_{2}+S_{3}+S_{4} .
$$

Hence the TBR cross-section for recombination into $n l$ state becomes

$$
\sigma_{n l}=\int \frac{2 m}{\left|\boldsymbol{p}_{1}+\boldsymbol{p}_{2}\right|} \frac{2 \pi}{\hbar}\left|M_{f i}\right|^{2} \frac{\mathrm{d}^{3} \boldsymbol{p}_{1}^{\prime}}{(2 \pi)^{3}} \frac{\mathrm{d}^{3} \boldsymbol{P}}{(2 \pi)^{3}},
$$

where $p_{1}, p_{2}$ are the space parts of the momenta of the two incident electrons, and $\boldsymbol{p}_{1}^{\prime}$ and $\boldsymbol{P}$ are the space parts of the momenta of the scattered electron and final ion, respectively, and

$$
\begin{aligned}
& \left|M_{f i}\right|^{2}=\left(S_{1}+S_{2}+S_{3}+S_{4}\right)\left(S_{1}+S_{2}+S_{3}+S_{4}\right)^{*} \\
& \quad=S_{1} S_{1}^{*}+S_{2} S_{2}^{*}+S_{3} S_{3}^{*}+S_{4} S_{4}^{*} \\
& \quad+2\left(S_{1} S_{2}^{*}+S_{1} S_{3}^{*}+S_{1} S_{4}^{*}+S_{2} S_{3}^{*}+S_{2} S_{4}^{*}+S_{3} S_{4}^{*}\right)
\end{aligned}
$$

where $S_{1} S_{2}^{*}=S_{2} S_{1}^{*}$ and so on. Calculation of the terms in the right-hand side of Eq. (25) are shown in the following subsections.

\subsubsection{Coefficient $S_{1} S_{1}^{*}$}

From (19) using Eqs. (2)-(14) we get

$$
S_{1}=C_{1} T_{1} T_{2} T_{3} I_{S_{1}}
$$

where

$$
\begin{aligned}
C_{1} & =\frac{e^{2}}{\hbar c}\left(\sqrt{\frac{1}{V(2 \pi)^{3}}}\right) \sqrt[6]{\frac{m^{2}}{E_{p_{1}^{\prime}} E_{p_{1}}}} \sqrt{\frac{m^{2} M^{2}}{E_{b} E_{p_{2}} E_{P} E_{Q}}} \frac{(2 \pi)^{4}}{(2 \pi)^{4}} \frac{2 \pi}{\sqrt{p_{1} p_{2}}} \\
T_{1} & =\left[\bar{u}\left(p_{1}^{\prime}\right) \gamma_{\mu} u\left(p_{1}\right)\right] \\
T_{2} & =\left[\bar{u}(b) \gamma_{\nu} u\left(p_{2}\right)\right], \\
T_{3} & =[\bar{U}(P) U(Q)]
\end{aligned}
$$

and the overlap integral

$$
\begin{aligned}
I_{S_{1}}= & \int \frac{\mathrm{e}^{\mathrm{i} q_{1}\left(r_{2}-r_{1}\right)}}{q_{1}^{2}} \mathrm{e}^{-\mathrm{i} p_{1}^{\prime} r_{1}} \mathrm{e}^{\mathrm{i} p_{1} r_{1}} \mathrm{e}^{-\mathrm{i} b r_{2}} \mathrm{e}^{\mathrm{i} p_{2} r_{2}} \\
& \times \mathrm{e}^{-\mathrm{i} P R} \mathrm{e}^{\mathrm{i} Q R} \phi_{n l}(x) \mathrm{d}^{4} q_{1} \mathrm{~d}^{4} r_{1} \mathrm{~d}^{4} r_{2} \mathrm{~d}^{4} R .
\end{aligned}
$$

The energy $\delta$-function $\delta\left(E_{i}-E_{f}\right)$ is obtained after equal time-integration for which $r_{10}=r_{20}=R_{0}=t$. The momentum $\delta$-function $\delta^{3}\left(\boldsymbol{P}_{i}-\boldsymbol{P}_{f}\right)$ is obtained after integration over $\boldsymbol{R}$. Integration over $r_{1}$ gives

$$
q_{1}=p_{1}-p_{1}^{\prime}
$$


The overlap integral (31) on using (9) becomes

$$
I_{S_{1}}=\delta\left(E_{i}-E_{f}\right) \delta^{3}\left(\boldsymbol{P}_{i}-\boldsymbol{P}_{f}\right)\left(p_{1}-p_{1}^{\prime}\right)^{-2} I_{1}
$$

where

$$
I_{1}=\int \mathrm{e}^{-\mathrm{i} x \cdot\left(p_{1}-\boldsymbol{p}_{1}^{\prime}+p_{2}\right)} \phi_{n l}(x) \mathrm{d}^{3} x .
$$

From Eq. (26) the product of $S_{1}$ and its conjugate $S_{1}^{*}$ is

$$
S_{1} S_{1}^{*}=C_{1}^{2}\left(T_{1} T_{1}^{*}\right)\left(T_{2} T_{2}^{*}\right)\left(T_{3} T_{3}^{*}\right) I_{S_{1}}^{2} .
$$

Calculation of the integral $I_{1}$ for recombination into the ground state and that of the trace parts are shown in Eqs. $(2 \mathrm{~A})$ and $(6 \mathrm{~A})-(8 \mathrm{~A})$ of Appendix.

\subsubsection{Coefficient $S_{2} S_{2}^{*}$}

Substituting Eqs. (2)-(14) in (20),

$$
S_{2}=C_{2} T_{1} T_{2}^{\prime \prime} T_{3}^{\prime} I_{S_{2}},
$$

where

$$
\begin{aligned}
C_{2} & =\frac{-Z e^{2}}{\hbar c}\left(\sqrt{\frac{1}{V(2 \pi)^{3}}}\right)^{6} \sqrt{\frac{m^{2}}{E_{p_{1}^{\prime}} E_{p_{1}}}} \sqrt{\frac{m^{2} M^{2}}{E_{b} E_{p_{2}} E_{P} E_{Q}}} \frac{2 \pi}{\sqrt{p_{1} p_{2}}}, \\
T_{2}^{\prime} & =\left[\bar{U}(P) \gamma_{\nu} U(Q)\right] \\
T_{3}^{\prime} & =\left[\bar{u}(b) u\left(p_{2}\right)\right],
\end{aligned}
$$

and $T_{1}$ is given in Eq. (28).

The overlap integral

$$
\begin{aligned}
I_{S_{2}}= & \int \frac{\mathrm{e}^{\mathrm{i} q_{1}\left(R-r_{1}\right)}}{q_{1}^{2}} \mathrm{e}^{-\mathrm{i} p_{1}^{\prime} r_{1}} \mathrm{e}^{\mathrm{i} p_{1} r_{1}} \mathrm{e}^{-\mathrm{i} b r_{2}} \mathrm{e}^{\mathrm{i} p_{2} r_{2}} \\
& \times \mathrm{e}^{-\mathrm{i} P R} \mathrm{e}^{\mathrm{i} Q R} \phi_{n l}(x) \mathrm{d}^{4} q_{1} \mathrm{~d}^{4} r_{1} \mathrm{~d}^{4} r_{2} \mathrm{~d}^{4} R .
\end{aligned}
$$

On using (9) and making equal-time integration and integration over $\boldsymbol{R}$ in (39) we get

$$
I_{S_{2}}=\delta\left(E_{i}-E_{f}\right) \delta^{3}\left(\boldsymbol{P}_{i}-\boldsymbol{P}_{f}\right)\left(p_{1}-p_{1}^{\prime}\right)^{-2} I_{2},
$$

where

$$
I_{2}=\int \mathrm{e}^{-\mathrm{i} \boldsymbol{x} \cdot \boldsymbol{p}_{2}} \phi_{n l}(x) \mathrm{d}^{3} \boldsymbol{x} .
$$

From (35) we get

$$
S_{2} S_{2}^{*}=C_{2}^{2}\left(T_{1} T_{1}^{*}\right)\left(T_{2}^{\prime} T_{2}^{\prime *}\right)\left(T_{3}^{\prime} T_{3}^{\prime *}\right) I_{S_{2}}^{2}
$$

Calculation of the trace parts and the integration $I_{2}$ for $1 s$ state are shown in Eqs. (6A), (9A), (10A), and (3A). 


\subsubsection{Coefficient $S_{3} S_{3}^{*}$}

From (21) using the definitions as given in Sec. 2.1 we get (Fig. 1c):

$$
S_{3}=C_{3} T_{1} T_{2}^{\prime \prime} T_{3}^{\prime \prime} I_{S_{3}}
$$

where

$$
\begin{aligned}
C_{3}= & \frac{-Z e^{4}}{\hbar^{2} c^{2}}\left(\sqrt{\frac{1}{V(2 \pi)^{3}}}\right)^{6} \sqrt{\frac{m^{2}}{E_{p_{1}^{\prime}} E_{p_{1}}}} \sqrt{\frac{m^{2} M^{2}}{E_{b} E_{p_{2}} E_{P} E_{Q}}} \\
& \times(2 \pi)^{8}\left[\frac{1}{(2 \pi)^{4}}\right]^{3} \frac{2 \pi}{\sqrt{p_{1} p_{2}}}, \\
T_{2}^{\prime \prime}= & {\left[\bar{u}(b) \gamma_{\nu}(\widehat{s}+m) \gamma_{\delta} u\left(p_{2}\right)\right], } \\
T_{3}^{\prime \prime}= & {\left[\bar{U}(P) \gamma_{\rho} U(Q)\right], }
\end{aligned}
$$

and $T_{1}$ is given in Eq. (28). The overlap integral

$$
\begin{aligned}
I_{S_{3}}= & \int \frac{\mathrm{e}^{\mathrm{i} q_{1}\left(r_{2}-r_{1}\right)}}{q_{1}^{2}} \mathrm{e}^{-\mathrm{i} p_{1}^{\prime} r_{1}} \mathrm{e}^{\mathrm{i} p_{1} r_{1}} \mathrm{e}^{-\mathrm{i} b r_{3}} \mathrm{e}^{\mathrm{i} p_{2} r_{2}} \frac{\mathrm{e}^{\mathrm{i} s\left(r_{3}-r_{2}\right)}(\widehat{s}+m)}{s^{2}-m^{2}} \frac{\mathrm{e}^{\mathrm{i} q_{2}\left(R-r_{3}\right)}}{q_{2}^{2}} \\
& \times \mathrm{e}^{-\mathrm{i} P R} \mathrm{e}^{\mathrm{i} Q R} \phi_{n l}(y) \mathrm{d}^{4} q_{1} \mathrm{~d}^{4} q_{2} \mathrm{~d}^{4} s \mathrm{~d}^{4} R \mathrm{~d}^{4} r_{1} \mathrm{~d}^{4} r_{2} \mathrm{~d}^{4} r_{3},
\end{aligned}
$$

where

$$
y=r_{3}-\boldsymbol{R},
$$

and $r_{3}$ is the position vector of the electron (Fig. 1c). Integrating over $r_{2}$ we get

$$
s=p_{1}-p_{1}^{\prime}+p_{2} .
$$

From the Feynman diagram (Fig. 1c)

$$
\boldsymbol{q}_{2}=\boldsymbol{P}-\boldsymbol{Q} .
$$

After substituting Eq. (47) in (46) and performing equal-time integration and integrating over $\boldsymbol{R}$ we get

$$
I_{S_{3}}=\delta\left(E_{i}-E_{f}\right) \delta^{3}\left(\boldsymbol{P}_{i}-\boldsymbol{P}_{f}\right)\left(p_{1}-p_{1}^{\prime}\right)^{-2} P^{-2}\left[\left(p_{1}-p_{1}^{\prime}+p_{2}\right)^{2}-m^{2}\right]^{-2} I_{3},
$$

where

$$
I_{3}=\int \mathrm{e}^{-\mathrm{i} y \cdot\left(s-q_{2}\right)} \phi_{n l}(y) \mathrm{d}^{3} y .
$$

The result of integration for recombination in the ground state is shown in Appendix (Eq. (4A)). Using (42) we get

$$
S_{3} S_{3}^{*}=C_{3}^{2}\left(T_{1} T_{1}^{*}\right)\left(T_{2}^{\prime \prime} T_{2}^{\prime \prime *}\right)\left(T_{3}^{\prime \prime} T_{3}^{\prime \prime *}\right) I_{S_{3}}^{2} .
$$

The traces are calculated in Eqs. (6A), (11A) and (12A) of Appendix. 


\subsubsection{Coefficient $S_{4} S_{4}^{*}$}

From (22) using the definitions in Sec. 2.1 we get

$$
S_{4}=C_{4} T_{1} t_{2} t_{3} I_{S_{4}},
$$

where

$$
\begin{aligned}
C_{4}= & \frac{Z e^{4}}{\hbar^{2} c^{2}}\left(\sqrt{\frac{1}{V(2 \pi)^{3}}}\right)^{6} \sqrt{\frac{m^{2}}{E_{p_{1}^{\prime}} E_{p_{1}}}} \sqrt{\frac{m^{2} M^{2}}{E_{b} E_{p_{2}} E_{P} E_{Q}}}(2 \pi)^{8} \\
& \times\left[\frac{1}{(2 \pi)^{4}}\right]^{3} \frac{2 \pi}{\sqrt{p_{1} p_{2}}}, \\
t_{2}= & {\left[\bar{U}(P) \gamma_{\nu}(\widehat{l}+M) \gamma_{\delta} U(Q)\right], } \\
t_{3}= & {\left[\bar{u}(b) \gamma_{\rho} u\left(p_{2}\right)\right], }
\end{aligned}
$$

and $T_{1}$ is as in (28). The overlap integral

$$
\begin{aligned}
I_{S_{4}} & =\int \frac{\mathrm{e}^{\mathrm{i} q_{1}\left(R-r_{1}\right)}}{q_{1}^{2}} \mathrm{e}^{-\mathrm{i} p_{1}^{\prime} r_{1}} \mathrm{e}^{\mathrm{i} p_{1} r_{1}} \mathrm{e}^{-\mathrm{i} b r_{2}} \mathrm{e}^{\mathrm{i} p_{2} r_{2}} \frac{\mathrm{e}^{\mathrm{i} l\left(R^{\prime}-R\right)}(\hat{l}+M)}{l^{2}-M^{2}} \frac{\mathrm{e}^{\mathrm{i} q_{2}^{\prime}\left(r_{2}-R^{\prime}\right)}}{q_{2}^{\prime 2}} \\
& \times \mathrm{e}^{-\mathrm{i} P R^{\prime}} \mathrm{e}^{\mathrm{i} Q R} \phi_{n l}(y) \mathrm{d}^{4} q_{1} \mathrm{~d}^{4} q_{2}^{\prime} \mathrm{d}^{4} l \mathrm{~d}^{4} R \mathrm{~d}^{4} R^{\prime} \mathrm{d}^{4} r_{1} \mathrm{~d}^{4} r_{2},
\end{aligned}
$$

where (Fig. 1d)

$$
y=r_{2}-\boldsymbol{R}^{\prime}
$$

and

$$
q_{2}^{\prime}=p_{1}-p_{1}^{\prime}-P .
$$

Integrating over $R$ we get

$$
l=p_{1}-p_{1}^{\prime}+Q
$$

Substituting for $r_{2}$ from (57) in (56) and making equal-time integration and integration over $\boldsymbol{R}$ we obtain

$$
\begin{aligned}
I_{S_{4}}= & \delta\left(E_{i}-E_{f}\right) \delta^{3}\left(\boldsymbol{P}_{i}-\boldsymbol{P}_{f}\right) \\
& \times\left(p_{1}-p_{1}^{\prime}\right)^{-2}\left(\boldsymbol{p}_{1}-\boldsymbol{p}_{1}^{\prime}-\boldsymbol{P}\right)^{-2}\left[\left(p_{1}-p_{1}^{\prime}+Q\right)^{2}-M^{2}\right]^{-2} I_{4},
\end{aligned}
$$

where $I_{4}=\int \mathrm{e}^{-\mathrm{i} \boldsymbol{y} \cdot\left(\boldsymbol{q}_{2}^{\prime}+\boldsymbol{p}_{2}\right)} \phi_{n l}(y) \mathrm{d}^{3} y$, which for recombination into $1 s$ state is shown in Appendix (Eq. (5A)).

From (52) the product of $S_{4}$ and its conjugate $S_{4}^{*}$ becomes

$$
S_{4} S_{4}^{*}=C_{4}^{2}\left(T_{1} T_{1}^{*}\right)\left(t_{2} t_{2}^{*}\right)\left(t_{3} t_{3}^{*}\right) I_{S_{4}}^{2} .
$$

The trace parts are shown in Eqs. (6A), (13A), and (14A). 


\subsubsection{Interference terms}

Using (26), (35), (42) and (52) we get

$$
\begin{aligned}
& S_{1} S_{2}^{*}=C_{1} C_{2} I_{S_{1}} I_{S_{2}}\left(T_{1} T_{1}^{*}\right)\left(T_{2} T_{3}^{\prime *}\right)\left(T_{3} T_{2}^{\prime *}\right), \\
& S_{1} S_{3}^{*}=C_{1} C_{3} I_{S_{1}} I_{S_{3}}\left(T_{1} T_{1}^{*}\right)\left(T_{2} T_{2}^{\prime \prime *}\right)\left(T_{3} T_{3}^{\prime \prime *}\right), \\
& S_{1} S_{4}^{*}=C_{1} C_{4} I_{S_{1}} I_{S_{4}}\left(T_{1} T_{1}^{*}\right)\left(T_{2} t_{3}^{*}\right)\left(T_{3} t_{2}^{*}\right), \\
& S_{2} S_{3}^{*}=C_{2} C_{3} I_{S_{2}} I_{S_{3}}\left(T_{1} T_{1}^{*}\right)\left(T_{2}^{\prime} T_{3}^{\prime \prime *}\right)\left(T_{3}^{\prime} T_{2}^{\prime \prime *}\right), \\
& S_{2} S_{4}^{*}=C_{2} C_{4} I_{S_{2}} I_{S_{4}}\left(T_{1} T_{1}^{*}\right)\left(T_{2}^{\prime} t_{2}^{*}\right)\left(T_{3}^{\prime} t_{3}^{*}\right), \\
& S_{3} S_{4}^{*}=C_{3} C_{4} I_{S_{3}} I_{S_{4}}\left(T_{1} T_{1}^{*}\right)\left(T_{2}^{\prime \prime} t_{3}^{*}\right)\left(T_{3}^{\prime \prime} t_{2}^{*}\right),
\end{aligned}
$$

Respective traces are calculated in Appendix.

\subsection{Recombination rate}

To obtain the TBR rate coefficient $\alpha_{n l}$ for hydrogen formation in $n l$ state at near-zero temperature plasma environment, we consider Maxwellian distribution of velocity $v$ :

$$
\alpha_{n l}=\left\langle\sigma_{n l} v\right\rangle
$$

where $\sigma_{n l}$ is the cross-section as given in $\mathrm{Eq}$. (24). The average is performed over Maxwellian distribution function $f(v)$, where

$$
f(v)=\left(\frac{m}{2 \pi k T}\right)^{3 / 2} \exp \left(-\frac{m v^{2}}{2 \pi k T}\right)
$$

$T$ is the absolute temperature and $k$ is the Boltzmann constant. With the two electrons involved in TBR the rate becomes

$$
\alpha_{n l}=\sigma_{n l} v[f(v)]^{2}
$$

and the total rate is

$$
\alpha_{\mathrm{T}}=\sum_{n, l} \alpha_{n l}
$$

\section{Results and discussions}

Present results for TBR rate coefficients (RC) $\alpha_{n l}$ and recombination cross-sections (CS) $\sigma_{n l}$ of proton with electron in presence of an observer electron at $300 \mathrm{~K}$ into the orbital states $n=1$ to 10 , are shown in Figs. 2 and 3 , 


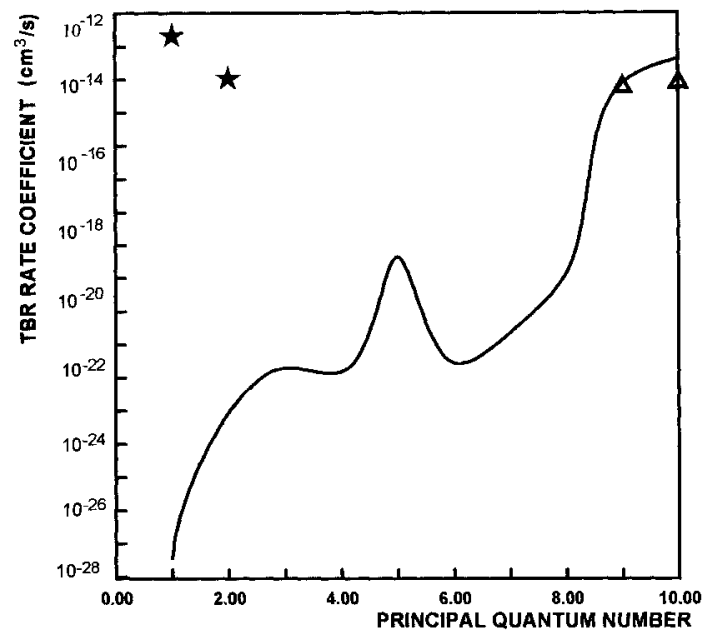

Fig. 2. Rate coefficient vs. principal quantum number for formation of H-atom at $300 \mathrm{~K}$. Continuous line represents present TBR results, open triangles represent theoretical TBR results of Stevefelt et al. [11] for formation of deuterium. Stars represent radiative recombination rate $[5 \mathrm{~b}]$.

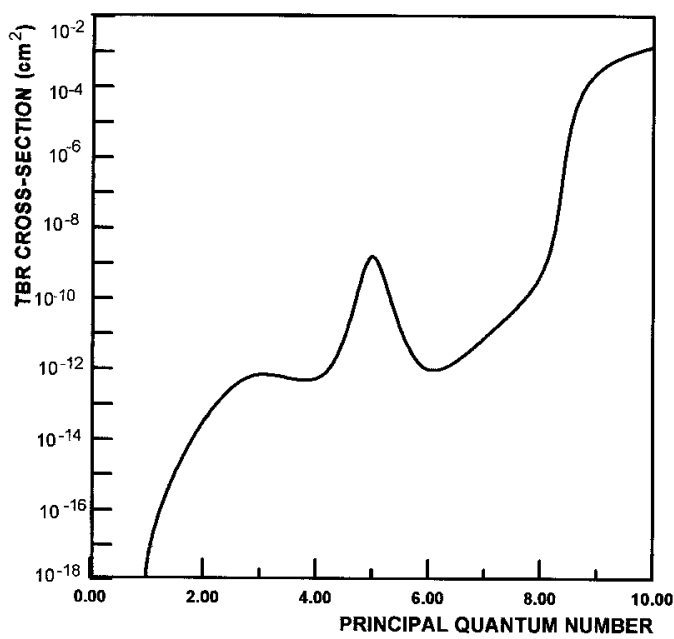

Fig. 3. Present result for cross-section for three-body recombination vs. principal quantum number for formation of $\mathrm{H}$-atom at $300 \mathrm{~K}$.

respectively. In the numerical calculation the Coulomb distortion to the plane wave (3) for low temperature reactions [5a, b] is approximated by Sommerfeld factor $\sqrt{2 \pi \eta_{\mu}}$.

Although our formulation for TBR is true for all $l$ states, we have done numerical calculation for capture into $s$ states only. The overlap integrals (33), $(40),(50)$, and (60) determine the contribution from different $l$ states. We found in 
[5a, b] that contributions from these type of integrals rapidly fall with the increase in $l$ value. Thus our knowledge from previous work on radiative recombination suggests that capture into the $s$ state dominates the process as compared to the capture into the higher $l$ states.

The present formulation for TBR is true for all the $n$ states. In Fig. 2 we have shown $\mathrm{RC}$ for the states $n=1$ to 10 where results are meagre. From our previous knowledge we know that $\mathrm{RR}$ rate, where photon takes away the extra energy, decreases [5a, b] rapidly with the increase in orbital quantum number $n$ while the present TBR rate, where extra energy is carried away by a third-body electron, increases with $n$. RR rate for recombination into atomic states 1 and 2 from our previous calculation [5b] lies between $10^{-12} \mathrm{~cm}^{3} / \mathrm{s}$ and $10^{-13} \mathrm{~cm}^{3} / \mathrm{s}$ (Fig. 2), while the corresponding TBR rates are respectively of the order $10^{-28} \mathrm{~cm}^{3} / \mathrm{s}$ and $10^{-26} \mathrm{~cm}^{3} / \mathrm{s}$. The TBR rate, however, increases to the order $10^{-12} \mathrm{~cm}^{3} / \mathrm{s}$ for $n=10$. The results of Stevefelt et al. [11] for TBR of electron with deuteron into 9 th and 10th orbits are shown in Fig. 2. It is to note that in the present work TBR has peaks, one flat and one sharp (Figs. 2, 3) for recombination into 3rd and 5 th orbits. The present results for $\mathrm{RC}$ at 5 th and 6 th orbits are respectively $10^{-18} \mathrm{~cm}^{3} / \mathrm{s}$ and $10^{-22} \mathrm{~cm}^{3} / \mathrm{s}$. Above $n=6$ there is a monotonic rise of $\mathrm{RC}$ until $n=8$, beyond which there is a steep rise of the curves till $n=9$. From $n=9$ to $n=10$ the rate of increase in $\mathrm{RC}$ is slow, being from $8 \times 10^{-14} \mathrm{~cm}^{3} / \mathrm{s}$ to $5 \times 10^{-13} \mathrm{~cm}^{3} / \mathrm{s}$. Results of Stevefelt et al. for TBR of deuterium into 9 th and 10 th orbits are closer to the present result for TBR as compared to the results by Bates et al. [10]. A decrease in RC (Fig. 4) and CS (Fig. 5) with temperature

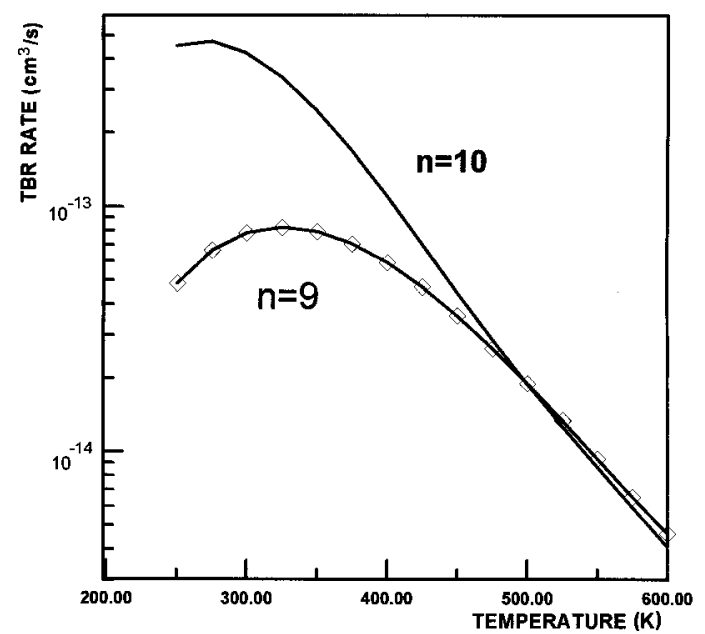

Fig. 4. Rate coefficient for three-body recombination vs. temperature for principal quantum numbers 9 and 10 . 


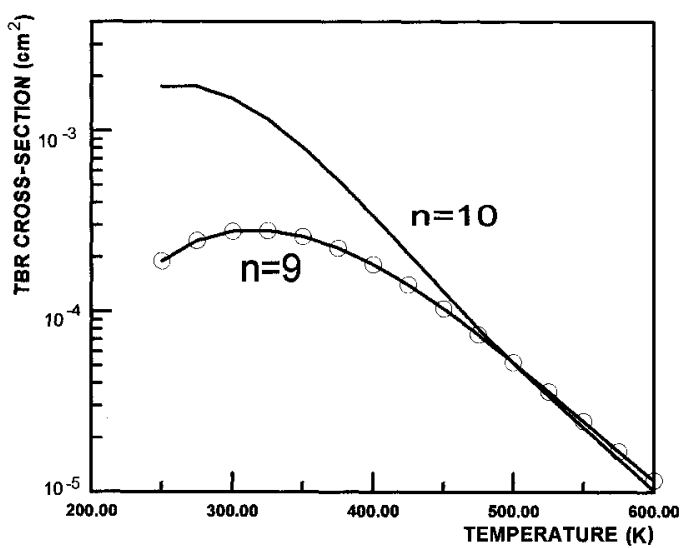

Fig. 5. Cross-section for three-body recombination vs. temperature for principal quantum numbers 9 and 10 .

for recombination into 9 th and 10 th orbits is found. Any difference between the present values and Stevefelt results may be due to mass difference of the isotopes.

Of all the four Feynman diagrams the maximum contribution comes from second-order diagrams (Figs. 1a and b) and the contribution from fourth-order Feynman diagram (Fig. 1d) is the lowest of all the states. We find that most of the contribution comes from SI process and contribution from higher-order TS process is several order smaller compared to SI process. Contribution from the interference of SI and TS processes is quite appreciable. TBR of antiparticles can be obtained in antiproton-positron plasma for the reaction

$$
\mathrm{p}^{-}+\mathrm{e}^{+}+\mathrm{e}^{+} \rightarrow \overline{\mathrm{H}}+\mathrm{e}^{+},
$$

$\overline{\mathrm{H}}$ and $\mathrm{e}^{+}$being the antihydrogen and positron, respectively. From symmetry consideration in QED, the Feynman diagrams with antiparticles will yield similar results in antiparticle plasma as the results in particle plasma. The present result agrees in principle with the results of Stevefelt et al. [11].

\section{Conclusion}

In conclusion we find it most encouraging that this compact yet simple formulation gives a good physical description of TBR process. The Feynman diagrams give some idea about the physics behind the recombination process. Formulation of this typical atomic collision problem in a gauge invariant language, which can describe most of the fundamental processes in nature, has its own intrinsic appeal. A comparative study between radiative recombination and three-body recombination shows that the RR decreases with the increase in orbital quantum number $n$ while TBR increases. Although we have considered TBR recombination into $s$ 
states of low orbits $(n=1,10)$ we hope to work on higher $n l$ states in our subsequent work. Finally, more experimental work is necessary on TBR in the low-lying states to verify our results.

\section{Appendix}

Calculation of the integrals $I_{i}$ in (33), (39), (46), and (56) for recombination into the ground state of hydrogenic ions.

The wave function of the $1 s$ ground state is

$$
\phi_{1 s}(x)=N \mathrm{e}^{-\alpha x},
$$

where $\alpha=\frac{Z}{a_{0}}, N=\left(\frac{Z}{a_{0}}\right)^{3 / 2} \frac{1}{\sqrt{\pi}}$ and for recombination into $1 s$ state

$$
\begin{aligned}
& I_{1}=\frac{N 8 \pi \alpha}{\left[\alpha^{2}+\left(\boldsymbol{p}_{1}-\boldsymbol{p}_{1}^{\prime}+\boldsymbol{p}_{2}\right)^{2}\right]^{2}}, \\
& I_{2}=\frac{N 8 \pi \alpha}{\left(\alpha^{2}+\boldsymbol{p}_{2}^{2}\right)^{2}}, \\
& I_{3}=\frac{N 8 \pi \alpha}{\left[\alpha^{2}+\left(\boldsymbol{p}_{1}-\boldsymbol{p}_{1}^{\prime}+\boldsymbol{p}_{2}-\boldsymbol{P}^{2}\right]^{2}\right.},
\end{aligned}
$$

and

$$
I_{4}=\frac{N 8 \pi \alpha}{\left[\alpha^{2}+\left(\boldsymbol{p}_{1}-\boldsymbol{p}_{1}^{\prime}-\boldsymbol{P}+\boldsymbol{p}_{2}\right)^{2}\right]^{2}} .
$$

The above integrals are also calculated with the help of [26] for recombinations into orbital quantum numbers $n=2$ to 10 .

Following the trace formulae in [25] and using Eqs. (28)-(30); (37), (38); and (44), (45) for the spinors we obtain for the trace parts the following results:

$$
\begin{aligned}
& T_{1} T_{1}^{*}=\frac{1}{4 m^{2}}\left[-8\left(p_{10}^{\prime} p_{10}-p_{1}^{\prime} \cdot p_{1}\right)+16 m^{2}\right], \\
& T_{2} T_{2}^{*}=\frac{1}{4 m^{2}}\left(-8 b_{0} p_{20}+16 m^{2}\right), \\
& T_{3} T_{3}^{*}=\frac{1}{4 M^{2}}\left(4 P_{0} Q_{0}+4 M^{2}\right), \\
& T_{2} T_{2}^{\prime *}=\frac{1}{4 M^{2}}\left(-8 P_{0} Q_{0}+16 M^{2}\right), \\
& T_{3} T_{3}^{\prime *}=\frac{1}{4 m^{2}}\left(4 b_{0} p_{20}+4 m^{2}\right),
\end{aligned}
$$




$$
\begin{aligned}
& T_{2}^{\prime \prime} T_{2}^{\prime \prime *}=\frac{16}{4 m^{2}}\left[b_{0} p_{20} s_{0}^{2}-2 b_{0} s_{0}\left(p_{2} \cdot s\right)+b_{0} p_{20}\left(s^{2}\right)-4 m^{2} b_{0} s_{0}+m^{2} b_{0} p_{20}\right. \\
& \left.\quad-4 m^{2} p_{20} s_{0}+4 m^{2}\left(p_{2} \cdot s\right)+4 m^{2} s_{0}^{2}-4 m^{2}\left(s^{2}\right)+4 m^{4}\right] \\
& T_{3}^{\prime \prime} T_{3}^{\prime \prime *}=\frac{1}{4 M^{2}}\left(-8 P_{0} Q_{0}+16 M^{2}\right) \\
& t_{2} t_{2}^{*}=\frac{16}{4 M^{2}}\left[P_{0} Q_{0} l_{0}^{2}-2 Q_{0} l_{0}(\boldsymbol{P} \cdot \boldsymbol{l})+P_{0} Q_{0}\left(l^{2}\right)-4 M^{2} P_{0} l_{0}+4 M^{2}(\boldsymbol{P} \cdot l)\right. \\
& \left.\quad-4 M^{2} Q_{0} l_{0}+M^{2} P_{0} Q_{0}+4 M^{2} l_{0}^{2}-4 M^{2}\left(l^{2}\right)+4 M^{4}\right] \\
& t_{3} t_{3}^{*}=\frac{1}{4 m^{2}}\left(-8 b_{0} p_{20}+16 m^{2}\right) \\
& T_{2} T_{3}^{\prime *}=\frac{1}{m}\left(b_{\alpha} g_{\alpha \nu}+p_{2 \beta} g_{\beta \nu}\right) \\
& T_{3} T_{2}^{\prime *}=\frac{1}{M}\left(P_{\gamma} g_{\gamma \nu}+Q_{\eta} g_{\eta \nu}\right)
\end{aligned}
$$

Taking the product of $(15 \mathrm{~A})$ and $(16 \mathrm{~A})$ we get

$$
\begin{aligned}
& \left(T_{2} T_{3}^{\prime *}\right) \times\left(T_{3} T_{22}^{\prime *}\right) \\
& \quad=\frac{1}{m M}\left[b_{0} P_{0}+b_{0} Q_{0}+p_{20} P_{0}-\left(p_{2} \cdot \boldsymbol{P}\right)+p_{20} Q_{0}\right] .
\end{aligned}
$$

Similarly, we obtain the following traces after some lengthy calculations:

$$
\begin{aligned}
& \left(T_{2} T_{2}^{\prime \prime *}\right) \times\left(T_{3} T_{3}^{\prime \prime *}\right)=\frac{2}{M m^{2}}\left[-b_{0} s_{0} p_{20} P_{0}-b_{0} s_{0} p_{20} Q_{0}-m^{2}\left(b_{0} P_{0}+b_{0} Q_{0}\right)\right. \\
& \quad+2 m^{2}\left(p_{20} P_{0}+p_{20} Q_{0}+s_{0} P_{0}+s_{0} Q_{0}\right)+\left(p_{2} \cdot \boldsymbol{P}\right)\left(b_{0} s_{0}-2 m^{2}\right) \\
& \left.\quad+(\boldsymbol{s} \cdot \boldsymbol{P})\left(b_{0} p_{20}-2 m^{2}\right)+\left(s \cdot p_{2}\right)\left(b_{0} P_{0}+b_{0} Q_{0}\right)\right] \\
& \left(T_{2} t_{3}^{*}\right) \times\left(T_{3} t_{2}^{*}\right)=\frac{1}{M m^{2}}\left[b_{0} l_{0} p_{20} P_{0}+b_{0} l_{0} p_{20} Q_{0}\right. \\
& \quad+b_{0} p_{20} P_{0} Q_{0}+M^{2} b_{0} p_{20}+m^{2}\left(P_{0} Q_{0}+P_{0} l_{0}+Q_{0} l_{0}\right)-2 b_{0} l_{0}\left(p_{2} \cdot \boldsymbol{P}\right) \\
& \left.\quad-2\left(\boldsymbol{p}_{2} \cdot l\right)\left(b_{0} P_{0}+b_{0} Q_{0}\right)+(\boldsymbol{P} \cdot l)\left(3 b_{0} p_{20}-m^{2}\right)+m^{2} M^{2}\right] \\
& \left(T_{2}^{\prime} T_{3}^{\prime \prime *}\right) \times\left(T_{3}^{\prime} T_{2}^{\prime \prime *}\right)=\frac{1}{M^{2} m}\left[P_{0} Q_{0} s_{0} b_{0}+P_{0} Q_{0} s_{0} p_{20}+P_{0} Q_{0} b_{0} p_{20}\right. \\
& \quad+m^{2} P_{0} Q_{0}+M^{2}\left(b_{0} s_{0}+b_{0} p_{20}+p_{20} s_{0}\right)-2(\boldsymbol{P} \cdot \boldsymbol{s})\left(Q_{0} b_{0}+Q_{0} p_{20}\right) \\
& \left.\quad-2 Q_{0} s_{0}\left(\boldsymbol{P} \cdot \boldsymbol{p}_{2}\right)+\left(\boldsymbol{p}_{2} \cdot \boldsymbol{s}\right)\left(3 P_{0} Q_{0}-M^{2}\right)+M^{2} m^{2}\right]
\end{aligned}
$$




$$
\begin{aligned}
& \left(T_{2}^{\prime} t_{2}^{*}\right) \times\left(T_{3}^{\prime} t_{3}^{*}\right)=\frac{2}{M^{2} m}\left[-P_{0} Q_{0} l_{0} b_{0}-P_{0} Q_{0} l_{0} p_{20}-M^{2}\left(P_{0} b_{0}+P_{0} p_{20}\right)\right. \\
& +2 M^{2}\left(Q_{0} b_{0}+Q_{0} p_{20}+l_{0} b_{0}+l_{0} p_{20}\right)+(\boldsymbol{P} \cdot l)\left(Q_{0} b_{0}+Q_{0} p_{20}\right) \\
& \left.+\left(\boldsymbol{l} \cdot \boldsymbol{p}_{2}\right)\left(P_{0} Q_{0}-2 M^{2}\right)+\left(\boldsymbol{P} \cdot \boldsymbol{p}_{2}\right)\left(-l_{0} Q_{0}+M^{2}\right)\right] \\
& \left(T_{2}^{\prime \prime} t_{3}^{*}\right) \times\left(T_{3}^{\prime \prime} t_{2}^{*}\right)=\frac{1}{m^{2} M^{2}}\left\{b_{0} l_{0} s_{0} Q_{0} p_{20} P_{0}+5\left[b_{0} l_{0} P_{0} Q_{0}\left(\boldsymbol{s} \cdot p_{2}\right)\right.\right. \\
& -b_{0} l_{0} s_{0} Q_{0}\left(\boldsymbol{p}_{2} \cdot \boldsymbol{P}\right)-P_{0} Q_{0} b_{0} s_{0}\left(\boldsymbol{p}_{2} \cdot \boldsymbol{l}\right)-p_{20} Q_{0} b_{0} l_{0}(\boldsymbol{s} \cdot \boldsymbol{P}) \\
& \left.+b_{0} Q_{0} s_{0} p_{20}(\boldsymbol{P} \cdot \boldsymbol{l})\right]-b_{0} Q_{0} p_{20} P_{0}(\boldsymbol{s} \cdot \boldsymbol{l})+b_{0} Q_{0}\left[2(\boldsymbol{s} \cdot \boldsymbol{l})\left(\boldsymbol{p}_{2} \cdot \boldsymbol{P}\right)\right. \\
& \left.+2(s \cdot \boldsymbol{P})\left(p_{2} \cdot l\right)+(\boldsymbol{P} \cdot \boldsymbol{l})\left(\boldsymbol{s} \cdot \boldsymbol{p}_{2}\right)\right]+M^{2}\left[p_{20} Q_{0} b_{0} s_{0}+b_{0} P_{0} s_{0} p_{20}\right. \\
& +b_{0} p_{20} s_{0} l_{0}+\left(s \cdot p_{2}\right)\left(-3 b_{0} P_{0}+b_{0} Q_{0}+b_{0} l_{0}\right)-3(s \cdot l) b_{0} p_{20} \\
& \left.+\left(\boldsymbol{p}_{2} \cdot \boldsymbol{P}\right) b_{0} s_{0}+\left(\boldsymbol{p}_{2} \cdot \boldsymbol{l}\right) b_{0} s_{0}+(\boldsymbol{s} \cdot \boldsymbol{P}) b_{0} p_{20}\right]+m^{2}\left[P_{0} Q_{0} b_{0} l_{0}\right. \\
& +P_{0} Q_{0} s_{0} l_{0}+P_{0} Q_{0} p_{20} l_{0}+(\boldsymbol{P} \cdot \boldsymbol{l})\left(-3 p_{20} Q_{0}+b_{0} Q_{0}+s_{0} Q_{0}\right) \\
& \left.-3 P_{0} Q_{0}(s \cdot l)+Q_{0} l_{0}(s \cdot \boldsymbol{P})+P_{0} Q_{0}\left(\boldsymbol{p}_{2} \cdot \boldsymbol{l}\right)+Q_{0} l_{0}\left(\boldsymbol{p}_{2} \cdot \boldsymbol{P}\right)\right] \\
& +m^{2} M^{2}\left[b_{0} P_{0}+b_{0} Q_{0}+b_{0} l_{0}+s_{0} P_{0}+s_{0} Q_{0}+s_{0} l_{0}+p_{20} P_{0}\right. \\
& \left.\left.+p_{20} Q_{0}+p_{20} l_{0}-(\boldsymbol{s} \cdot \boldsymbol{P})-(\boldsymbol{s} \cdot \boldsymbol{l})-\left(\boldsymbol{p}_{2} \cdot \boldsymbol{P}\right)-\left(\boldsymbol{p}_{2} \cdot \boldsymbol{l}\right)\right]\right\} .
\end{aligned}
$$

\section{Acknowledgment}

This work is supported by UGC, New Delhi, through project No. F10-17/98 (SR-I).

\section{References}

[1] H.S.W. Massey, E.H.S. Burhop, Electronic and Ionic Impact Phenomena, Clarendon Press, Oxford 1952.

[2] D.R. Bates, A.E. Kingston, Nature 189, 652 (1961).

[3] R.W.P. McWhirter, Nature 190, 902 (1961).

[4] N. D’Angelo, Phys. Rev. 121, 505 (1961).

[5] (a) S. Bhattacharyya, Acta Phys. Pol. A 86, 297 (1994); (b) S. Bhattacharyya, A. Roy, S. Mitra, Fizika A 6, 149 (1997).

[6] S. Bhattacharyya, A. Roy, Acta Phys. Pol. A 95, 911 (1999). 
[7] G. Gabrielse, S.L. Rolston, L. Haarsma, W. Kells, Hyperfine Interact. 44, 287 (1988).

[8] M. Charlton, J. Eades, D. Horvath, R.J. Hughes, C. Zimmermann, Phys. Rep. 241, 65 (1994).

[9] H.F. Beyer, D. Liesen, O. Guzman, Part. Accel. 24, 163 (1989).

[10] D.R. Bates, A.E. Kingston, R.W.P. McWhirter, Proc. R. Soc. A 267, 297 (1962).

[11] J. Stevefelt, J. Boulmer, J.F. Delpech, Phys. Rev. A 12, 1246 (1975).

[12] L.H. Andersen, J. Bolko, P. Kvistgaard, Phys. Rev. Lett. 64, 729 (1994).

[13] A. Muller, S. Schennach, M. Wagner, J. Haselbauer, O. Uwira, W. Spies, E. Jennewein, R. Becker, M. Kleinod, U. Probstel, N. Angert, J. Klabunde, P.H. Mokler, P. Spadtke, B. Wolf, Phys. Scr. Vol. T 37, 62 (1991).

[14] A. Muller, S. Schennach, M. Wagner, J. Haselbauer, O. Uwira, W. Spies, E. Jennewein, R. Becker, M. Kleinod, U. Probstel, N. Angert, J. Klabunde, P.H. Mokler, P. Spadtke, B. Wolf, Z. Phys. D 21, S205 (1991).

[15] A. Frank, A. Muller, S. Schennach, M. Wagner, J. Haselbauer, O. Uwira, W. Spies, E. Jennewein, R. Becker, M. Kleinod, N. Angert, P.H. Mokler, in: Atomic Physics of Highly-Charged Ions, Eds. P. Richard, M. Stockli, C.L. Cocke, C.D. Lin, American Institute of Physics, New York 1993, p. 532.

[16] A. Wolf, J. Berger, M. Bock, D. Habs, B. Hochadel, G. Kilgus, G. Neureither, U. Schramm, D. Schwalm, E. Szmola, A. Muller, M. Wagner, R. Schuch, Z. Phys. $D$ 21, S69 (1991).

[17] T. Quinteros, H. Gao, D.R. Dewitt, R. Schuch, M. Pajek, S. Asp, Dz. Belkic, Phys. Rev. A 51, 1340 (1995).

[18] Y. Hahn, P. Kristic, J. Phys. B, At. Mol. Opt. Phys. 27, L509 (1994).

[19] C. Biedermann, H. Gao, R. Schuch, W. Zong, S. Asp, D.R. Dewitt, H. Kuiper, J. Phys. B, At. Mol. Opt. Phys. 28, 505 (1995).

[20] S. Bhattacharyya, K. Pathak, Phys. Scr. 54, 143 (1996).

[21] S. Bhattacharyya, K. Pathak, Indian J. Phys. B 74, 455 (2000).

[22] S. Bhattacharyya, S. Mitra, Phys. Rev. A 60, 2269 (1999); 62, 032709 (2000).

[23] A.L. Akhiezer, V.B. Berestetsky, Quantum Electrodynamics, Interscience, New York 1965, Ch. 4, p. 5.

[24] S. Bhattacharyya, K. Rinn, E. Salzborn, L. Chatterjee, J. Phys. B 21, 111 (1988).

[25] J.D. Bjorken, S.D. Driell, Relativistic Quantum Mechanics, McGraw-Hill, USA 1964.

[26] Handbook of Mathematical Functions, Eds. M. Abramowitz, I.A. Stegun, Dover, New York 1964, p. 508. 